\title{
An 80 au cavity in the disk around HD 34282
}

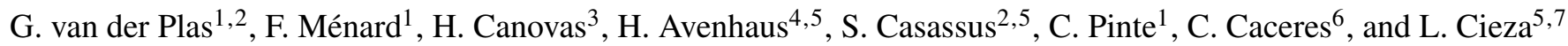 \\ ${ }^{1}$ Univ. Grenoble Alpes, CNRS, IPAG (UMR 5274), 38000 Grenoble, France \\ e-mail: info@gerritvanderplas.com \\ 2 Departamento de Astronomía, Universidad de Chile, Casilla 36-D, Santiago, Chile \\ 3 Departamento de Física Teórica, Universidad Autónoma de Madrid, Cantoblanco, 28049 Madrid, Spain \\ ${ }^{4}$ ETH Zurich, Institute for Astronomy, Wolfgang-PauliStrasse 27, 8093 Zurich, Switzerland \\ 5 Millenium Nucleus Protoplanetary Disks in ALMA Early Science, Universidad de Chile, Casilla 36-D, Santiago, Chile \\ ${ }^{6}$ Departamento de Ciencias Físicas, Facultad de Ciencias Exactas, Universidad Andrés Bello, Av. Fernàndez Concha 700, \\ Las Condes, Santiago, Chile \\ 7 Nucleo de Astronomia, Facultad de Ingeniería, Universidad Diego Portales, Av. Ejércíto 441, Santiago, Chile
}

Received 18 June 2017 / Accepted 5 July 2017

\begin{abstract}
Context. Large cavities in disks are important testing grounds for the mechanisms proposed to drive disk evolution and dispersion, such as dynamical clearing by planets and photoevaporation.

Aims. We aim to resolve the large cavity in the disk around HD 34282, whose presence has been predicted by previous studies modeling the spectral energy distribution of the disk.

Methods. Using ALMA band 7 observations we studied HD 34282 with a spatial resolution of $0.10^{\prime \prime} \times 0.17^{\prime \prime}$ at $345 \mathrm{GHz}$.

Results. We resolve the disk around HD 34282 into a ring between $0.24^{\prime \prime}$ and $1.15^{\prime \prime}\left(78_{-11}^{+7}\right.$ and $374_{-54}^{+33}$ au adopting a distance of $325_{-47}^{+29} \mathrm{pc}$ ). The emission in this ring shows azimuthal asymmetry centered at a radial distance of $0.46^{\prime \prime}$ and a position angle of $135^{\circ}$ and an azimuthal FWHM of $51^{\circ}$. We detect $\mathrm{CO}$ emission both inside the disk cavity and as far out as 2.7 times the radial extent of the dust emission.

Conclusions. Both the large disk cavity and the azimuthal structure in the disk around HD 34282 can be explained by the presence of a $50 M_{\text {jup }}$ brown dwarf companion at a separation of $\approx 0.1^{\prime \prime}$.
\end{abstract}

Key words. stars: variables: $\mathrm{T}$ Tauri, Herbig $\mathrm{Ae} / \mathrm{Be}$ - protoplanetary disks

\section{Introduction}

Protoplanetary disks are the birth environments of planetary systems. How these planets form is an ongoing topic of debate that is informed by an increasing number of disks that show various degrees of evolution and dispersal, such as opacity cavities (transitional disks), gaps (pre-transitional disks, e.g. Williams \& Cieza 2011), and asymmetrical (lopsided) emission features. Examples of such disks imaged at (sub) mm wavelengths include HD 100546 (Walsh et al. 2014), Sz 91 (Canovas et al. 2015, 2016), HD 142527 (Casassus et al. 2013), HD 97048 (van der Plas et al. 2017) and SAO 206462 (Brown et al. 2009).

The common denominator between these disks is that their structure can be described by one large cavity or a broad ring of dust grains at reasonably large radii combined with multiple rings, gaps and/or azimuthal horseshoe-shaped asymmetries in the outer disk. The gaps and/or cavities in these disks are not empty: they contain both smaller dust grains, as traced by scattered light imaging (e.g., Kraus \& Ireland 2012; Avenhaus et al. 2014), and gas, as most readily traced by rotational (Perez et al. 2015a; van der Marel et al. 2015) and rovibrational carbon monoxide $(\mathrm{CO})$ lines (van der Plas et al. 2009, 2015; Pontoppidan et al. 2011; Carmona et al. 2014). Recently, long baseline Atacama Large Millimeter Array (ALMA) observations of HL Tau (ALMA Partnership et al. 2015),
HD 163296 (Isella et al. 2016), and TW Hya (Andrews et al. 2016; Nomura et al. 2016; Tsukagoshi et al. 2016) have demonstrated that these disks show a rich substructure of many concentric rings and gaps at scales as small as 1 au when observed at very high spatial resolution. It is indeed possible that most disks contain similar detailed structures that have not yet been resolved (Zhang et al. 2016).

Radial and azimuthal asymmetries in the dust emission structure of disks reflects variations in either the underlying mass, grain properties (size distribution), and/or temperature. The response of the disk to local pressure maxima is a frequently invoked mechanism to explain these structures. A local maximum in the gas density preferentially traps larger grains and thus stops the inward drift motion caused by the aerodynamic drag of the gas on the dust (Weidenschilling 1977), allowing dust grains to accumulate in these so-called dust traps. Such local gas pressure maxima have many proposed origins; for example, the maxima are generated at the edge of a dead zone (Dzyurkevich et al. 2010), by MRI instabilities (Uribe et al. 2011), or at the edge of a planet-carved gap (e.g. Pinilla et al. 2012). These maxima can even form spontaneously in simulations when the growth and fragmentation of dust grains and the back reaction of the dust grains on the gas is taken into account (Gonzalez et al. 2017). Jumps in the pressure gradient can also induce vortices due to Rossby wave instability (RWI, e.g. Lovelace et al. 1999; Lyra \& Lin 2013). Such vortices are especially promising places 
Table 1. Details of the observations.

\begin{tabular}{lllllll}
\hline \hline UT date & $\begin{array}{l}\text { Number } \\
\text { antennas }\end{array}$ & $\begin{array}{l}\text { Baseline range } \\
(\mathrm{m})\end{array}$ & $\begin{array}{l}p w v \\
(\mathrm{~mm})\end{array}$ & Flux & $\begin{array}{l}\text { Calibrators: } \\
\text { Bandpass }\end{array}$ & Gain \\
\hline 2014 Dec. 12 & 37 & 15.1 to 348.5 & 0.811 & J0423-013 & J0522-3627 & J0501-0159 \\
2015 Aug. 31 & 38 & 15.1 to 1466.2 & 0.755 & J0423-013 & J0423-0120 & J0542-0913 \\
\hline
\end{tabular}

for planetesimal formation given their high efficiency in concentrating large dust masses (Meheut et al. 2012). A RWI however is only stable in low viscosity disks with $\alpha \lesssim 10^{-4}$ (de Val-Borro et al. 2007). Another mechanism proposed to explain horseshoe-shaped features in disks is the presence of an unequal-mass binary companion. For large enough mass ratios the cavity becomes eccentric causing an azimuthally localized gas overdensity on the outer edge of the cavity (Ragusa et al. 2017).

Detection and characterization of more structured disks are necessary to determine the physical origin of these asymmetries. Disks around the intermediate mass Herbig Ae/Be (HAeBe) stars are good candidates for spatially resolving such structures, given their higher brightness and larger size compared to disks around the more abundant but lower mass/luminosity $\mathrm{T}$ Tauri stars. These disks have historically been split into two groups based on the shape of their spectral energy distribution (SED). Group I sources display relatively bright mid- to far-infrared emission and have been interpreted as hosting gas-rich protoplanetary disks with a flared, bright dust surface. Most dust in group II disks is assumed to have settled toward the midplane and these disks therefore emit weaker mid- to far-infrared emission (Meeus et al. 2001; Dullemond \& Dominik 2004). Recent modeling of resolved observations of group I sources suggests that the bright infrared emission of these sources should instead be attributed to the large vertical walls on the limit of (large) dust cavities (Honda et al. 2012; Maaskant et al. 2013); this idea is supported by high resolution scattered light imaging (Garufi et al. 2017) and spatially resolved CO rovibrational observations (van der Plas et al. 2015).

In this manuscript we present ALMA observations of the group I disk around the Herbig Ae/Be star HD 34282. The disk around this star has already been resolved using $1.3 \mathrm{~mm}$ continuum emission with a FWHM of $1.74^{\prime \prime} \times 0.89^{\prime \prime}$ (Piétu et al. 2003) and with rotational CO lines (Greaves et al. 2000; Piétu et al. 2003). Acke et al. (2009) predicted the presence of an opacity cavity in this disk based on modeling the SED. Khalafinejad et al. (2016) used spatially resolved $Q$-band emission to estimate a gap that is $92(+31,-17)$ au in size.

Multiple estimates of the stellar parameters for HD 34282 exist. We use the values derived by Merín et al. (2004) that are based on detailed modeling of the stellar spectrum and the SED. These authors found low metal abundances in the stellar spectrum and derived an A3 V spectral type, an age of $6.4_{-2.6}^{+1.9} \mathrm{Myr}$, a luminosity of $13.64_{-12.02}^{+5.36} L_{\odot}$, and a mass of $1.59_{-0.07}^{+0.30} M_{\odot}$ for the central star. For the source distance we use the value of $325_{-47}^{+29} \mathrm{pc}$ from the Gaia DR1 (Gaia Collaboration 2016a,b). This value is within the $1 \sigma$ values on the distance determined by Merín et al. (2004) of $348_{-77}^{+129}$ and of $400_{-100}^{+170}$ pc (Piétu et al. 2003).

\section{Observations and data reduction}

ALMA Early Science Cycle 2 observations were conducted in the compact C43-2 configuration on December 12, 2014 with $684 \mathrm{~s}$ of total time on source and in an extended C34-7 configuration on August 31, 2015 with 342 s of total time on source. The array configuration provided baselines ranging between 15.1 and $348.5 \mathrm{~m}$ and between 15.1 and $1466.2 \mathrm{~m}$, respectively. During the observations the precipitable water vapor had a median value at zenith of 0.811 and $0.755 \mathrm{~mm}$, respectively.

Two of the four spectral windows of the ALMA correlator were configured in time division mode (TDM) to maximize the sensitivity for continuum observations (128 channels over $1.875 \mathrm{GHz}$ usable bandwidth). These two TDM spectral windows were centered at 345.8 and $356.7 \mathrm{GHz}$. The other two spectral windows were configured in frequency division mode (FDM) to target the ${ }^{12} \mathrm{CO} J=3-2$ and the $\mathrm{HCO}^{+} J=4-3$ lines with a spectral resolution of 105 and $103 \mathrm{~m} \mathrm{~s}^{-1}$, respectively, using $0.23 \mathrm{GHz}$ total bandwidth. The data were calibrated and combined using the Common Astronomy Software Applications pipeline (CASA, McMullin et al. 2007, version 4.5). Inspection of the calibrated visibilities shows a $20 \%$ difference in amplitude between the two observations at short baselines. Given that the estimated error on the flux calibrator for the extended array configuration observations is twice as large as that for the compact configuration observations and assuming that the emission from the midplane is constant in the eight month period spanning the observations, we scaled the amplitudes of the visibilities for the extended array configuration observations to those of the compact array configuration observations. Details of the observations and calibration are summarized in Table 1, we estimated the absolute flux calibration to be accurate within $\sim 20 \%$.

We imaged the disks with the CLEAN task in CASA (Högbom 1974) using Briggs and superuniform weighting, which results in a restored beam of $0.25^{\prime \prime} \times 0.19^{\prime \prime}$ at $\mathrm{PA}=88.3^{\circ}$ (Briggs) and $0.17^{\prime \prime} \times 0.10^{\prime \prime}$ at $\mathrm{PA}=77.0^{\circ}$ (superuniform), respectively. The dynamic range of these images is strongly limited by the bright continuum source and we performed selfcalibration on both phase and amplitude, resulting in a final rms of $0.11 \mathrm{mJy} / \mathrm{beam}$ for the images created using superuniform weighting. We show the resulting continuum map in Fig. 1. We applied the self-calibration solutions obtained from the continuum emission to the $\mathrm{HCO}^{+}$and $\mathrm{CO}$ calibrated visibilities and subtracted the continuum emission using the CASA task uvcon$t s u b$. The resulting integrated intensity (moment 0 ), intensityweighted mean velocity (moment 1 ) and peak intensity (moment 8) maps and spectra are shown in Figs. 2 and 3 for the $\mathrm{CO} J=3-2$ and $\mathrm{HCO}^{+} J=4-3$ emission, respectively.

\section{Results}

\subsection{Continuum}

The HD 34282 dust continuum emission is concentrated into a ring that shows an azimuthal variation in intensity (Fig. 1). We use the fitting library uvmultifit (Martí-Vidal et al. 2014) to fit a superposition of simple geometrical shapes to the continuum visibilities to estimate the inclination, position angle, and spatial distribution of the emission. The uvmultifit fitting library minimizes $\chi^{2}$ as a function of the input model parameters. Quoted 


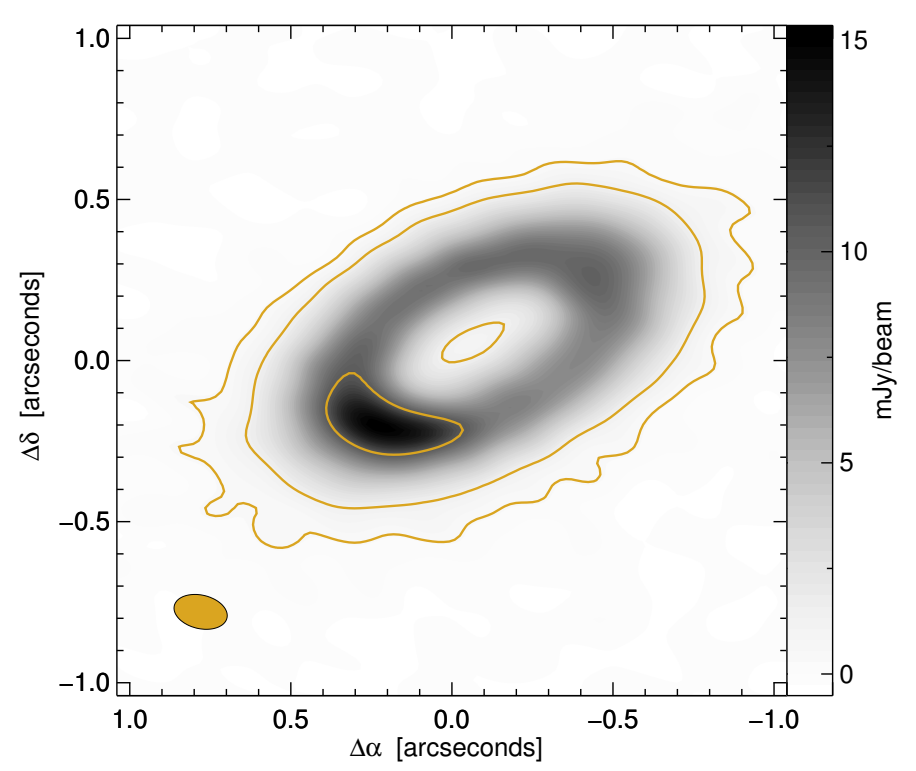

Fig. 1. Continuum image of HD 34282 for the ALMA band 7 (351.24 GHz or $0.853 \mathrm{~mm}$ ) observations, reconstructed using superuniform weighting resulting in a $0.10^{\prime \prime} \times 0.17^{\prime \prime}$ beam. Overplotted are contours at 5,15 and 100 times the rms value of $0.11 \mathrm{mJy} / \mathrm{beam}$. The beam is shown in orange in the bottom left.

parameter uncertainties are estimated from the post-fit covariance matrix which is scaled so that its $\chi_{r}^{2}$ has a value of 1 .

We fit the continuum visibilities to a combination of the following geometries: (1) a disk with a constant surface brightness; (2) an unresolved ring; and (3) a 2D Gaussian whose size and center are left free. Each of these elements has the following free parameters: offset in RA and Dec from the phase center, flux, semimajor axis, axis ratio, and position angle. The disk element has two values for the semimajor axis: one for the inner radius of the disk (the cavity radius) and one for the outer radius of the disk. When fitting two disks or a disk and a ring simultaneously we force them to share the same center, inclination, and position angle. We fit these shapes for each of the continuum windows (at 345.8 and $356.7 \mathrm{GHz}$ ) separately to allow the detection of possible changes in flux due to the spectral slope of the dust emission $\alpha\left(S_{v} \propto v^{\alpha}\right)$.

We achieve the best fit with a combination of two disk components and a Gaussian that is offset from the disk center, as judged by minimizing the residuals of the calibrated visibilities of the data and our models. The best-fit parameters for the fitted components are listed in Table 2 and are visualized in Fig. 4, in which we compare the imaged model and residuals to the HD 34282 disk, together with the real part of the visibilities for the data, the model, and their differences. Overall, our best fit provides a reasonable match to the data. The center of the cavity is in agreement with the stellar position published in the Gaia DR1 (Gaia Collaboration 2016a,b), and the dominant source of residuals is a radial pattern alternating between negative and positive signals that are most visible along the major axis of the disk. This pattern is a consequence of using disk models with a radial constant surface brightness and a sharp drop at the disk boundary. The most significant non-radially symmetric residuals are visible at the far side of the disk close to the disk minor axis, on the order of $1.0 \mathrm{mJy} /$ beam, nine times the image rms.

We start our analysis with the following values derived from our best fit: a disk with a total flux of $323.9 \mathrm{mJy}$ at $345.8 \mathrm{GHz}$
$(867 \mu \mathrm{m})$ with a spectral slope of $-3.0 \pm 0.7$ coming from a disk that extends between $0.24^{\prime \prime}$ and $0.94^{\prime \prime}$, an inclination of $59.3^{\circ}$ and a position angle of $117.1^{\circ}$.

\subsubsection{Radial and azimuthal structure of the dust emission}

From fitting the visibilities with a superposition of simple geometrical shapes we find that $\approx 95 \%$ of the continuum emission originates from a ring between 0.24 and $0.94^{\prime \prime}$ from the central star, while the remaining 5\% are concentrated in a region that is elongated in the azimuthal direction at a radial distance of $\approx 0.36^{\prime \prime}$ from the star atop the smooth disk. Looking at the imaged residuals (Fig. 4) there are hints of faint emission both inside the cavity and outside the fitted rings.

The emission within the cavity, also visible in the imaged residuals, indicates that there is at least some dust inside the fitted cavity radius at $0.24^{\prime \prime}$. The amplitude of this residual emission is comparable to that of the residuals at the inner and outer boundaries of the fitted disks, and is likely caused by our choice of fitting a disk with a sharp edge.

To better characterize the radial and azimuthal structure of the dust disk, we remapped the disk to polar coordinates after deprojecting it using the previously derived geometry (Fig. 5). The intensity profile in polar coordinates is shown in the top left panel and is collapsed in the radial (top right panel) and azimuthal (bottom left panel) dimensions to obtain the respective integrated surface brightness structures.

The radial brightness profile for the continuum emission rises monotonically until it peaks at $0.44^{\prime \prime}$. After that it decreases and shows a change in slope around 1.15" until it becomes indistinguishable from the background around $1.35^{\prime \prime}$, as shown in the bottom left panel of Fig. 5. The radial brightness distribution of the dust continuum emission between the peak at $0.44^{\prime \prime}$ and the outer disk radius at $1.35^{\prime \prime}$ can be fitted by two power laws separated at $1.15^{\prime \prime}$ with exponents of -6 and -16 , respectively.

The azimuthal brightness profile for the continuum emission peaks at an azimuth of $135^{\circ}$ with a maximum $\approx 25 \%$ above the median value for the disk and $18^{\circ}$ away from the major axis of the disk. To further study the azimuthal structure of the disk we subtract a median radial Gaussian profile from each azimuthal row in the polar projection. We create this average Gaussian by selecting only those radial slices with an integrated value within $1 \%$ of the median integrated value over all azimuths (Fig. 6). The remaining structure peaks close to the major axis of the disk and is best fit with a 2D Gaussian centered on (radius, azimuth) $=\left(0.43^{\prime \prime}, 18^{\circ}\right)$ with a FWHM of $0.15^{\prime \prime}$ in radius and $52^{\circ}$ in azimuth. This feature is discussed in Sect. 4.

\section{2. $\mathrm{CO}$ and $\mathrm{HCO}^{+}$emission}

We detect spatially and spectrally resolved emission from the ${ }^{12} \mathrm{CO} J=3-2$ and $\mathrm{HCO}^{+} J=4-3$ emission lines from the HD 34282 disk. We show the moment maps and line profiles in Figs. 2 and 3 respectively.

We estimate the systemic velocity from the ${ }^{12} \mathrm{CO} J=3-2$ emission line at $v_{\mathrm{LSR}}=-2.35 \pm 0.10 \mathrm{~km} \mathrm{~s}^{-1}$, based on the center of the line profile and channel maps. The line flux, integrated between + and $-6.75 \mathrm{~km} \mathrm{~s}^{-1}$ from the systemic velocity is $21.2 \pm 0.3 \mathrm{Jy} \mathrm{km} \mathrm{s}^{-1}$. The semimajor axis, as measured from the ${ }^{12} \mathrm{CO}$ moment maps is 3.1 ". We detect ${ }^{12} \mathrm{CO}$ emission coming from within the disk cavity and see two layers of $\mathrm{CO}$ emission on either side of the disk surface (Fig. 7), which shows that the ${ }^{12} \mathrm{CO}$ emission originates from the warm disk surface on both 

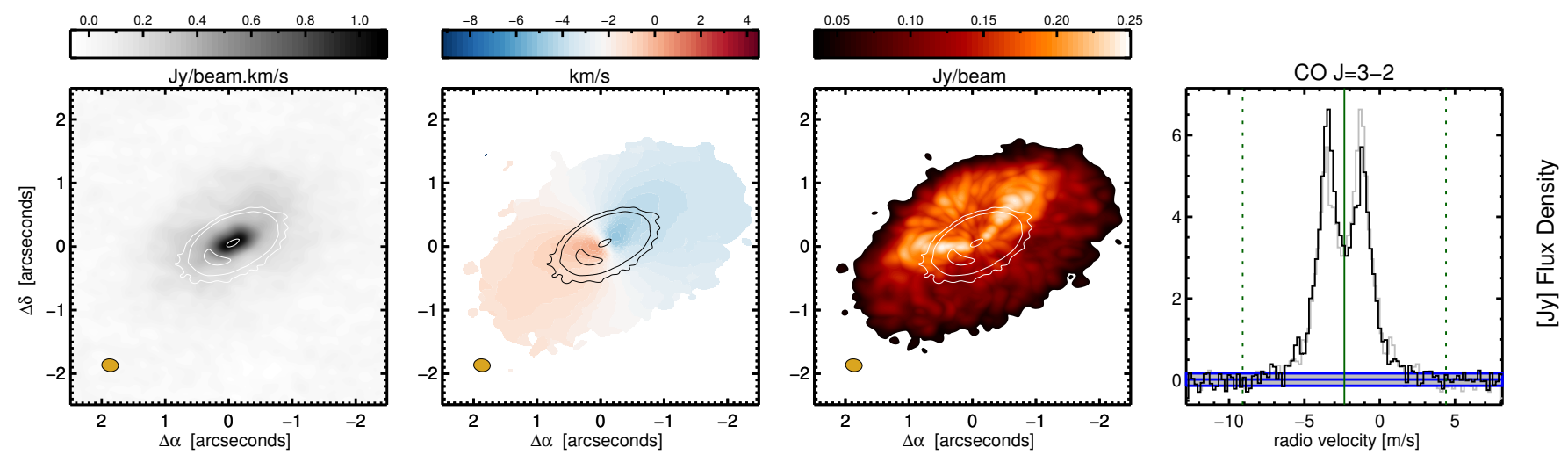

Fig. 2. Summary of ${ }^{12} \mathrm{CO} J=3-2$ line emission from the disk around HD 34282. We show the integrated intensity (moment 0 , left panel), intensity-weighted velocity (moment 1,2 nd panel), peak intensity (moment $8,3 \mathrm{rd}$ panel) and the collapsed emission line (right panel). Each moment map was made using a $5 \sigma$ cutoff and imaged using Briggs weighting. Overplotted in the three left panels are continuum contours with [5, 15 and 100] times the rms value of the continuum map imaged using superuniform weighting. The beam is shown in orange in the bottom left of each panel. The grayshaded area in the right panel denotes the + and $-3 \sigma$ level calculated outside the line boundaries.
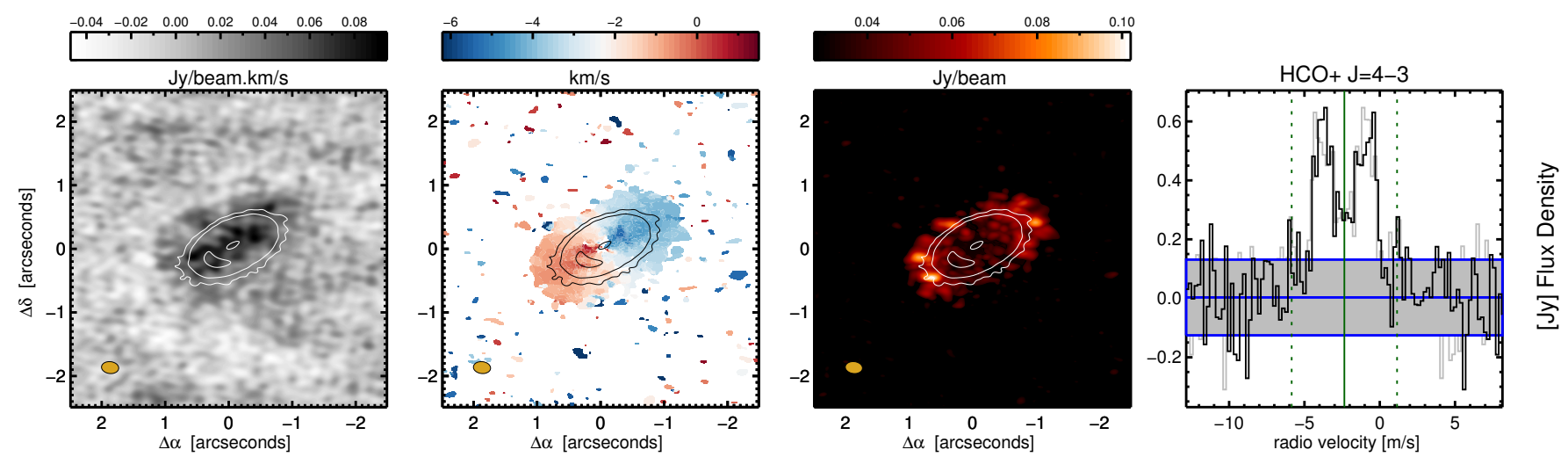

Fig. 3. Summary of $\mathrm{HCO}^{+} J=4-3$ line emission in HD 34282. We show the integrated intensity (moment 0 , left panel), intensity-weighted velocity (moment 1, 2nd panel), peak intensity (moment $8,3 \mathrm{rd} \mathrm{panel)}$ and the collapsed emission line (right panel). Each moment map was made using a $3 \sigma$ cutoff and imaged using Briggs weighting. Overplotted in the three left panels are continuum contours with [5, 15 and 100] times the rms value of the continuum emission. The beam is shown in orange in the bottom left of each panel. The grayshaded area in the right panel denotes the + and $-3 \sigma$ level calculated outside the line boundaries.

faces of the disk. We calculate an upper limit on the inner radius of the gas disk assuming Keplerian rotation for the highest velocity at which we detect the line above $3 \sigma$, and measure the radial outer extent of the gas disk based on the presence of emission above three times the rms value. We summarize the measured gas disk size and line flux in Table 3 . In the naturally weighted channel maps $\mathrm{CO}$ emission $>3 \sigma$ is present between -8.8 and $+4.6 \mathrm{~km} \mathrm{~s}^{-1}$. These values can be translated to an emitting radius of $\approx 23 \mathrm{au}$, assuming the gas is in Keplerian rotation in a disk inclined by $59.3^{\circ}$ around a $1.59 M_{\odot}$ star.

The low- $J{ }^{12} \mathrm{CO}$ rotational emission lines in protoplanetary disks become optically thick quickly and trace a vertically thin region in the line of sight up to where the line becomes optically thick, making them a tracer of disk geometry (e.g. de Gregorio-Monsalvo et al. 2013; Woitke et al. 2016). This makes it possible to infer the disk geometry and spatial extend from the ${ }^{12} \mathrm{CO} J=3-2$ moment 8 (peak intensity) map shown in Fig. 2, panel 3. Warmer $\mathrm{CO}$ gas emits stronger per unit volume, and the modulation of the peak brightness over the disk surface can be naturally interpreted as looking into a flared disk (bowl) where the far side of the disk is the warm and directly irradiated disk surface. This warm surface is shielded by the flaring outer disk on the near (south-west) side, from which we instead see the cooler midplane and the backside of the disk.

The $\mathrm{HCO}^{+}$emission is less extended with a semimajor axis of $1.3^{\prime \prime}$ and is, with a flux of $2.2 \pm 0.2 \mathrm{Jy} \mathrm{km} \mathrm{s}^{-1}, 10$ times weaker than the $\mathrm{CO}$ emission. Based on the resolved emission in the channel maps the emission comes from deeper in the disk (closer to the midplane) and emission $>3 \sigma$ is present between -5.8 and $+1.2 \mathrm{~km} \mathrm{~s}^{-1}$. These values can be translated to an emitting inner radius of $\approx 85 \mathrm{au}$, assuming the gas is in Keplerian rotation in a disk inclined by $59.3^{\circ}$ around a $1.59 M_{\odot}$ star. The $\mathrm{HCO}^{+}$is brightest beyond the outer radius of the continuum ring, as seen in the moment 8 map in Fig. 3. We confirm that this is an artifact of subtracting the continuum from the $\mathrm{HCO}^{+}$emission by repeating our analysis using the non-continuum-subtracted data. This suggests either that the continuum is absorbing part of the line emission mostly from the far side of the disk or that the $\mathrm{HCO}^{+}$emission is optically thick.

\section{Discussion}

In the following section we discuss the mass of the disk and its components, the spatial distribution of the dust and gas, and possible mechanisms that can provoke such a system architecture. 
G. van der Plas et al.: An 80 au cavity in the disk around HD 34282

Table 2. Best-fit parameters with their respective $1 \sigma$ uncertainty in parenthesis, obtained from fitting separate components to the continuum visibilities: either one component (radially constant disk, a ring, and a Gaussian) or a combination of these components.

\begin{tabular}{|c|c|c|c|c|c|c|c|c|}
\hline Component & $\begin{array}{l}\Delta \mathrm{RA}^{a} \\
{\left[{ }^{\prime \prime}\right]}\end{array}$ & $\begin{array}{l}\Delta \operatorname{Dec}^{a} \\
{\left[{ }^{\prime \prime}\right]}\end{array}$ & $\begin{array}{l}S_{v, 345.8 \mathrm{GHz}} \\
{[\mathrm{mJy}]}\end{array}$ & $\alpha$ & $\begin{array}{l}\text { Semimajor axis } \\
{\left[{ }^{\prime \prime}\right]}\end{array}$ & $\begin{array}{l}\text { Inclination } \\
{\left[{ }^{\circ}\right]}\end{array}$ & $\begin{array}{l}\mathrm{PA} \\
{\left[{ }^{\circ}\right]}\end{array}$ & $X_{r}^{2}$ \\
\hline \multicolumn{9}{|l|}{1 component } \\
\hline Gaussian (G) & -0.039 & 0.017 & $338.0(0.7)$ & $-3.2(0.1)$ & $0.53(0.01)$ & $60.4(0.4)$ & $119.4(0.4)$ & 9.69 \\
\hline Ring (R) & -0.046 & 0.022 & $312.3(0.5)$ & $-2.9(0.1)$ & $0.50(0.01)$ & $59.0(0.3)$ & $116.7(0.4)$ & 9.30 \\
\hline Disk (D) & -0.044 & 0.021 & $322.4(0.6)$ & $-3.1(0.1)$ & $0.19(0.07), 0.74(0.03)^{b}$ & $59.4(0.6)$ & $117.5(0.4)$ & 8.97 \\
\hline \multicolumn{9}{|c|}{$>1$ components } \\
\hline $\begin{array}{l}\mathrm{D}+\mathrm{R} \\
\text { Disk 1 } \\
\text { Ring 1 } \\
\text { Total }\end{array}$ & $\begin{array}{l}-0.053 \\
\text { fixed }\end{array}$ & $\begin{array}{l}0.029 \\
\text { fixed }\end{array}$ & $\begin{array}{l}252.6(1.3) \\
65.6(1.3) \\
318.2(1.8) \\
\end{array}$ & $\begin{array}{l}-3.2(0.2) \\
-1.6(0.9) \\
-2.9(0.4) \\
\end{array}$ & $\begin{array}{l}0.21(0.02), 0.75(0.01)^{b} \\
0.45(0.01)\end{array}$ & $\begin{array}{l}59.7(0.4) \\
\text { fixed }\end{array}$ & $\begin{array}{l}118.0(0.1) \\
\text { fixed }\end{array}$ & 5.76 \\
\hline $\begin{array}{l}\text { D + D } \\
\text { Disk 1 } \\
\text { Disk } 2 \\
\text { Total } \\
\end{array}$ & $\begin{array}{l}-0.053 \\
\text { fixed }\end{array}$ & $\begin{array}{l}0.029 \\
\text { fixed }\end{array}$ & $\begin{array}{l}215.1(2.1) \\
108.1(2.2) \\
323.1(3.0)\end{array}$ & $\begin{array}{l}-3.1(0.5) \\
-2.8(1.0) \\
-3.0(0.6)\end{array}$ & $\begin{array}{l}0.26(0.02), 0.61(0.01)^{b} \\
0.32(0.06), 0.90(0.01)\end{array}$ & $\begin{array}{l}59.6(0.4) \\
\text { fixed }\end{array}$ & $\begin{array}{l}117.9(0.3) \\
\text { fixed }\end{array}$ & 5.50 \\
\hline $\begin{array}{l}\text { D + D + G } \\
\text { Disk 1 } \\
\text { Disk 2 } \\
\text { Gaussian } \\
\text { Total }\end{array}$ & $\begin{array}{l}-0.063 \\
\text { fixed } \\
0.270^{c}\end{array}$ & $\begin{array}{l}0.039 \\
\text { fixed } \\
-0.240^{c}\end{array}$ & $\begin{array}{l}221.2(2.1) \\
87.0(2.1) \\
15.7(0.3) \\
323.9(3.0)\end{array}$ & $\begin{array}{l}-4.5(1.1) \\
-2.5(0.5) \\
-1.5(1.1) \\
-3.0(0.7)\end{array}$ & $\begin{array}{l}0.24(0.01), 0.63(0.01)^{b} \\
0.34(0.07), 0.94(0.01) \\
0.15(0.01)\end{array}$ & $\begin{array}{l}59.3(0.4) \\
\text { fixed } \\
70.1(1.0)\end{array}$ & $\begin{array}{l}117.1(0.3) \\
\text { fixed } \\
67.4(4.6)\end{array}$ & 5.41 \\
\hline
\end{tabular}

Notes. When fitting a disk and ring component simultaneously, the following parameters were fixed between the two: the offset from the pointing center, the inclination, and position angle. In the table we represent these values as "fixed". The spectral slope $\alpha$ (5th column) is calculated following $S_{v} \propto v^{\alpha}$. ${ }^{(a)}$ Offset from the pointing center. ${ }^{(b)}$ Contains two values for the disk component: the inner and outer radius. ${ }^{(c)}$ Offset relative to the center of the best-fit disk and ring component.
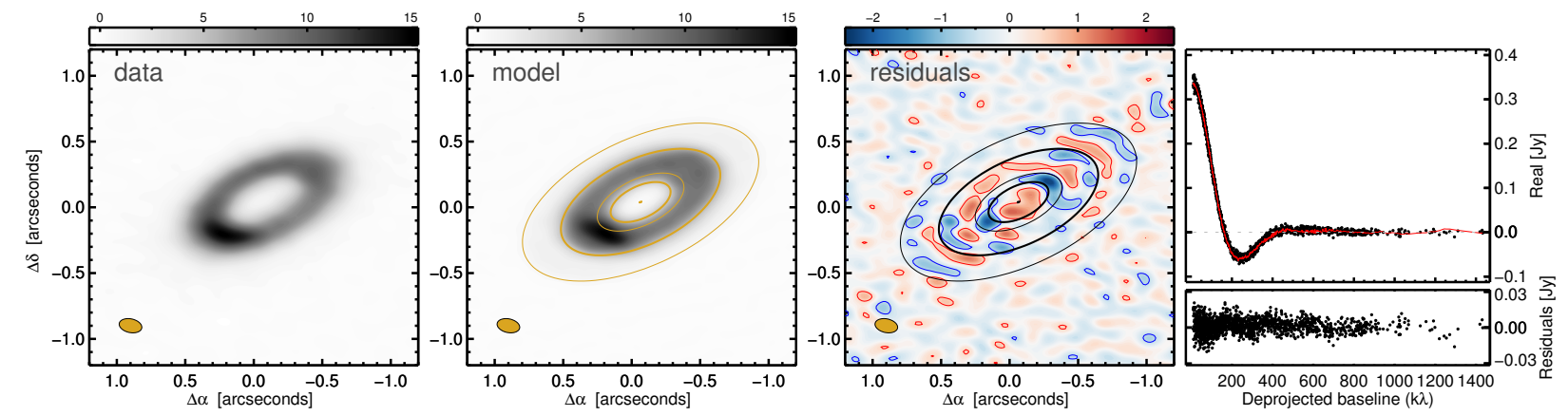

Fig. 4. Comparison of ALMA band 7 data (left panel) with the best-fit composite "disk + disk + Gaussian" model ( 2 nd panel). The $3 r d$ panel shows the imaged residuals. We draw red and blue contours at 5 times the image rms of $0.11 \mathrm{mJy}$. Units of all intensity scales are in $\mathrm{mJy} / \mathrm{beam}$. The central two panels include ellipses representing the 2 fitted disk components to guide the eye. These are drawn at the radial location corresponding to the inner and outer disk radius for disk \#1 (thick line) and disk \#2 (thin line), as summarized in Table 2. The right panel (top) shows the real part of the visibilities as function of the deprojected baseline for the data (black dots) and the model (red line). The bottom panel shows the residuals. The visibilities are binned in sets of 200 .

\subsection{Mass limits on the disk and vortex-shaped feature}

A coarse but straightforward way to relate observed sub-mm fluxes to dust mass is by assuming the emitting dust is optically thin and of a single (average) temperature,

$\log M_{\text {dust }}=\log S_{v}+2 \log d-\log \kappa_{v}-\log B_{v}\left(\left\langle T_{\text {dust }}\right\rangle\right)$,

where $S_{v}$ is the flux density, $d$ is the distance, $\kappa_{v}$ is the dust opacity, and $B_{v}\left(\left\langle T_{\text {dust }}\right\rangle\right)$ is the Planck function evaluated at the average dust temperature (Hildebrand 1983). We adopt a dust opacity of $2.7 \mathrm{~cm}^{2} \mathrm{~g}^{-1}$ at $0.867 \mathrm{~mm}$, calculated using smoothed UV astronomical silicate (Draine \& Lee 1984; Laor \& Draine 1993; Weingartner \& Draine 2000) with a grain size distribution with sizes between 0.1 and $3000 \mu \mathrm{m}$ distributed following a power law with a slope of -3.5 . We estimate the average dust temperature using the correlation with stellar luminosity $\left\langle T_{\text {dust }}\right\rangle \approx 25\left(L_{*} / L_{\odot}\right)^{1 / 4} \mathrm{~K}$ (e.g. Andrews et al. 2013), leading to a $\left\langle T_{\text {dust }}\right\rangle$ of $48 \mathrm{~K}$. This is most likely an overestimate of the dust temperature since all dust in the disk around HD 34282 is located outside $\approx 78$ au and the resulting dust mass should thus be interpreted as a lower limit. Using these assumptions, we calculate a dust mass of $0.41 M_{\text {jup }}$ for the disk and 6.6 $M_{\text {earth }}$ for the vortex-shaped feature. With a dust-to-gas ratio of 1:100 this leads to a disk that weights $2.5 \%$ of the stellar mass. The lineof-sight optical depth, which i calculated with the parameters of our best-fit disk model, a dust opacity of $2.7 \mathrm{~cm}^{2} \mathrm{~g}^{-1}$, and an albedo of 0.75 , is 1.1 in the vortex-shaped feature and 0.6 along the ring. 


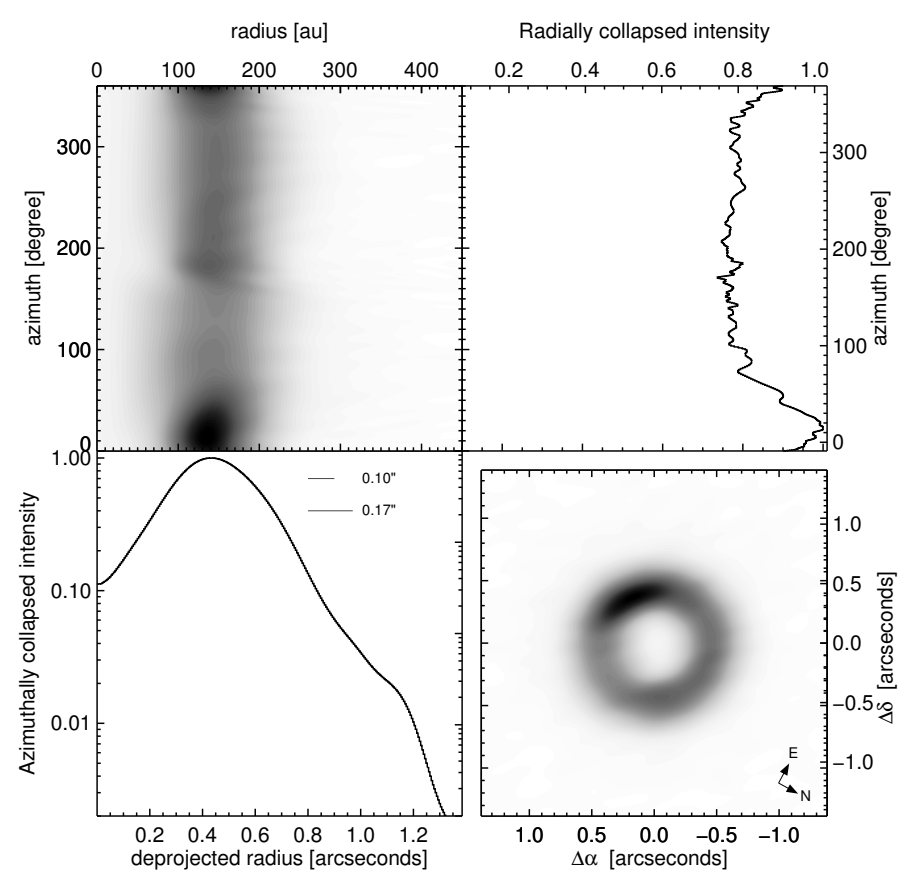

Fig. 5. ALMA band 7 image reconstructed using superuniform weighting, deprojected using the disk inclination and rotated with the position angle listed in Table 2 to put the major axis of the disk at $0^{\circ}$ (north; bottom right panel), converted to polar coordinates (top left panel). This polar map is collapsed along the radial and azimuthal axes to yield the azimuthal intensity distribution (top right) and the radial intensity distribution (bottom left). In this last panel we also show the size of the beam minor and major axis for reference.

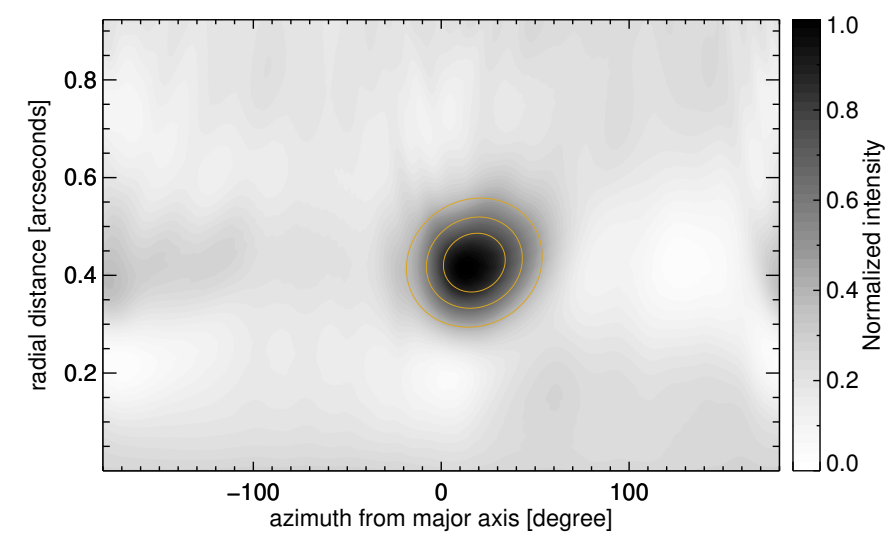

Fig. 6. Surface brightness of the disk after subtracting a Gaussian fit to the median radial profile, shown in polar coordinates with the position angle relative to the major axis of the disk on the $x$-axis and the radial distance from the center on the $y$-axis. Contours of the best-fit 2D Gaussian are show at values of $[0.25,0.5$ and 0.75$]$ times its peak value.

\subsection{Radial and azimuthal structure of the dust emission}

Both defining features of the dust continuum emission, the ring and the azimuthal asymmetry, can be explained by the dynamics of gas and dust at the edge of the cavity if there is an unseen massive companion present. Ragusa et al. (2017) use 3D SPH gas and dust simulations to test the effect of a binary pair of unequal mass on a circumbinary disk. In their simulations the companion carves a wide and eccentric cavity resulting in a nonaxisymmetric gas overdensity at the cavity edge. The amount of azimuthal asymmetry in their simulations scales with the binary mass ratio; a ratio of 0.01 results in a ring-like structure, while a mass ratio of 0.05 produces a contrast ratio of $\approx 1.5$ between the feature and background disk. Since the brightness contrast ratio in the HD 34282 disk is $\approx 1.25$, a naive interpolation would put the companion mass at $\approx 0.05 M_{\odot}\left(50 M_{\text {jup }}\right)$.

The presence of a binary companion has already been suggested by Wheelwright et al. (2010) based on the spectroastrometric signal of the $\mathrm{H} \beta$ line. No estimate of the separation or position angle is given, however, owing to artifacts in the astrometric signal. The spectro-astrometric technique used is sensitive to binaries with a separation between $0.1^{\prime \prime}$ and $2^{\prime \prime}$ with a brightness contrast of up to 5 mag (Baines et al. 2006), putting the companion somewhere between $0.1^{\prime \prime}$ and the cavity radius at $0.24^{\prime \prime}$. A separation close to $0.1^{\prime \prime}$ is in agreement with predictions by Artymowicz \& Lubow (1994) for the location of the cavity outer edge, who predict the inner edge for the circumbinary disk to be between 1.8 to 2.6 times the binary semimajor axis for low (between 0 and 0.25 ) eccentricities.

This scenario, where a brown dwarf binary companion induces the outer disk morphology, is backed up by the following arguments:

(1) Besides dynamical clearing, inside-out photoevaporation of the disk by the stellar radiation is often invoked as cause for disk cavities (e.g. Clarke et al. 2001; Alexander et al. 2006; Owen et al. 2010). However, in the case of this disk we deem photoevaporation unlikely to have shaped the cavity. The nondetection of $\mathrm{CO}$ gas close to the star in both the rotational (this work) and rovibrational lines (Carmona et al. 2005) is consistent with a photoevaporation scenario. However, an important caveat is the sensitivity of the latter CO observations. Typical line strengths for $\mathrm{CO}$ rovibrational lines originating from disks around $\mathrm{HAeBe}$ disks are about 5 to $10 \%$ above the continuum level (e.g. van der Plas et al. 2015). Such lines could easily be hidden in the noise given the spectrum presented in Fig. 1 of Carmona et al. (2005), which makes HD 34282 a prime target for high sensitivity observations of the fundamental rovibrational $\mathrm{CO}$ lines.

The arguments against photoevaporation as a cause for the cavity are stronger. Cavities carved out by radiation pressure from the central star are predicted to have a sharp edge, which is not the case for the HD 34282 disk. The insideout nature of photoevaporation as a root cause for the cavity also precludes the presence of any inner disk, while a halo or small inner disk is required to fit the near-infrared (NIR) excess observed for this source (Khalafinejad et al. 2016).

(2) The stellar Fe/H abundance for HD 34282 is strongly depleted; this is in line with the suggestion by Kama et al. (2015) that depletion of heavy elements emerges as companions block the accretion of part of the dust, while gas continues to flow toward the central star.

The spectral index value we derive for the complete disk $(-3.0 \pm 0.7)$ is lower than, but within uncertainty consistent with, the canonical value of $\approx-2.3$ for disks (Testi et al. 2014). This lower value is in line with the trend observed in, for example, AS 209, where the grain size distribution is weighted more toward larger grains in the inner disk leading to a value of $\alpha>-2.5$ at 20 au and increasing to $<-3.5$ outside 80 au (Pérez et al. 2012).

One out of the three components, the Gaussian offset from the disk center, in our best-fit disk model deviates $>1 \sigma$ from the typical value for young disks of -2.3 , whereas the small value of $\alpha$ in the fitted Gaussian suggests advanced grain growth. However, given the large error on this value we do not consider this deviation significant. Upcoming work on this source, comparing these data with $1.3 \mathrm{~mm}$ ALMA measurements at a similar 


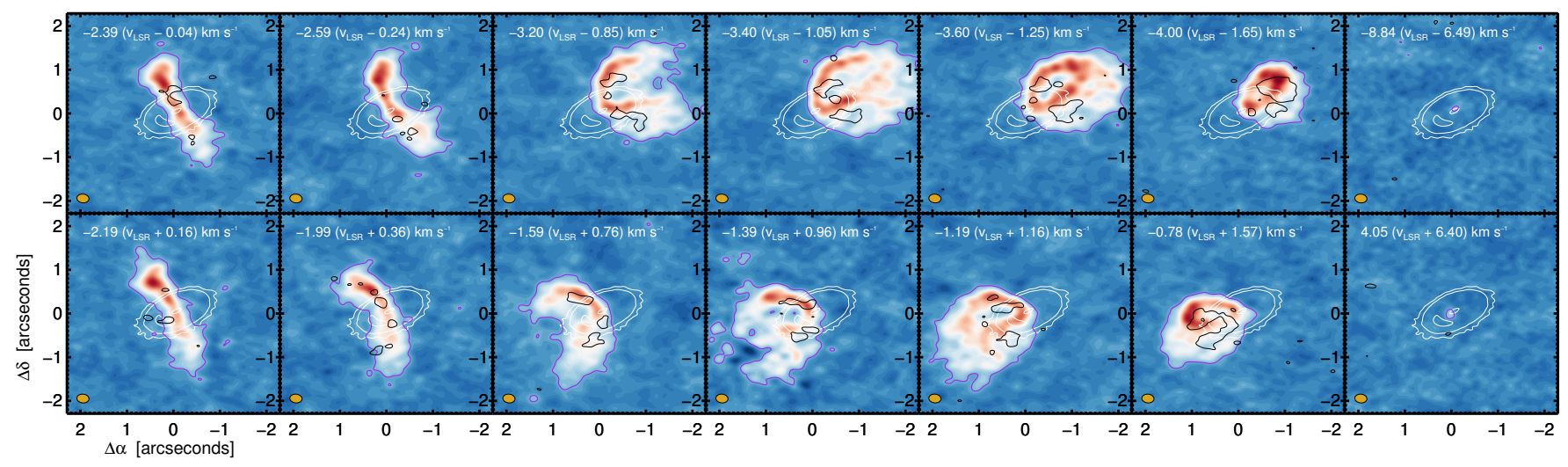

Fig. 7. Selected channel maps showing the $\mathrm{CO}$ channels in color and their $3 \sigma$ outline in purple, the $\mathrm{HCO}^{+}$emission $3 \sigma$ outline with black contours, and the continuum contours in white. In the top right of each panel the $v_{\mathrm{lsr}}$ is indicated in white noting the velocity with respect to the systemic velocity of $-2.35 \mathrm{~km} \mathrm{~s}^{-1}$ in parenthesis. The clean beam is shown in yellow in the bottom left of each panel.

Table 3. Line fluxes, spectral resolution and spatial extent for the $\mathrm{CO}$ $J=3-2$ and $\mathrm{HCO}^{+} J=4-3$ lines.

\begin{tabular}{lllllll}
\hline \hline Line & $\begin{array}{l}\text { Line flux } \\
\left(\mathrm{Jy} \mathrm{km} \mathrm{s}^{-1}\right)\end{array}$ & $\begin{array}{l}\text { Error }^{a} \\
\left(\mathrm{Jy} \mathrm{km} \mathrm{s}^{-1}\right)\end{array}$ & $\begin{array}{l}\text { Channel width } \\
\left(\mathrm{m} \mathrm{s}^{-1}\right)\end{array}$ & $\begin{array}{l}\mathrm{rms}^{b} \\
(\mathrm{mJy} / \text { beam })\end{array}$ & $\begin{array}{l}\text { Major axis } \\
{ }^{c}\end{array}$ & $\begin{array}{l}r_{\text {in }}{ }^{d} \\
(\mathrm{au})\end{array}$ \\
\hline $\mathrm{CO} J=3-2$ & 21.2 & 0.3 & 200 & 9.4 & 6.2 & 23 \\
$\mathrm{HCO}^{+} J=4-3$ & 2.2 & 0.2 & 200 & 10.4 & 2.6 & 85 \\
\hline
\end{tabular}

Notes. Line fluxes have been calculated from the Briggs-weighted images by integrating the emission around the systemic velocity at $-2.35 \mathrm{~km} \mathrm{~s}^{-1}$ assuming a half line width of $3.5 \mathrm{~km} \mathrm{~s}^{-1}$ for the $\mathrm{HCO}^{+}$ $J=4-3$ line and $6.75 \mathrm{~km} \mathrm{~s}^{-1}$ for the $\mathrm{CO} J=3-2$ line. ${ }^{(a)}$ Estimated from the rms of the integrated spectrum outside the line boundaries, does not include calibration uncertainties. ${ }^{(b)} 1 \sigma$ rms per channel. ${ }^{(c)} \mathrm{Ma}-$ jor axis is determined for all emission above 3 times the rms per channel. ${ }^{(d)}$ Calculated from the maximum velocity for which emission $>3 \sigma$ is present in the channel maps, assuming the gas is in Keplerian rotation in a disk inclined with $59.3^{\circ}$ around a $1.59 M_{\odot}$ star.

angular resolution, will be better able to decide the nature of the azimuthal brightness modulation. This azimuthally asymmetric feature in the disk originates from the same radial distance as the peak emission of the dust ring as can be seen in Fig. 6 . The radial size of the disk is comparable to the beam and thus could be unresolved. The feature is resolved in the azimuthal direction with a FWHM of $52^{\circ}$.

\subsection{Distribution and kinematics of the gas}

It is possible that $\mathrm{CO}$ gas is present closer to the star than the 24 au limit measured from our data because beam dilution would render the emission undetectable given the size of our beam $(65 \times 85$ au for the Briggs-weighted maps). In the outer disk regions the CO gas can be traced out as far as 3.1" (1000 au), which is a factor of $\approx 2.7$ further out than the dust detection coming from larger grains.

Judging by the velocity field, the $\mathrm{CO}$ emission is in agreement with that from a Keplerian rotating disk. We verify our choice for distance $(325 \mathrm{au})$ and stellar mass $\left(1.59 M_{\odot}\right)$ using kinematics of the $\mathrm{CO}$ emission. We construct a position-velocity map using the disk position derived from fitting the continuum emission and compare this with the expected Keplerian rotation curves for the stellar distance and mass derived by Piétu et al. (2003) and Merín et al. (2004), respectively, in Fig. 8. The inner radius for the $\mathrm{CO}$ rotation curves is set at 24 au and the outer radius is set either at 835 au (from Piétu et al. 2003) or at 1080 au.
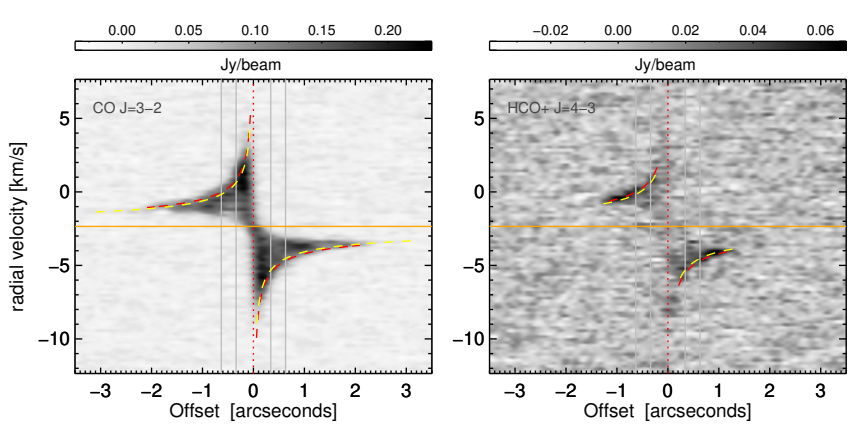

Fig. 8. Position-velocity diagram for the $\mathrm{CO} J=3-2(l e f t)$ and $\mathrm{HCO}^{+}$ $J=3-2$ (right) emission made using the disk center and position angle listed in Table 2. We use a dotted vertical red line to show the stellar position. The systemic velocity of $-2.35 \mathrm{~km} \mathrm{~s}^{-1}$ is shown with an orange horizontal line. We show the Keplerian rotation curves for the two different estimates for stellar mass and distance from Merín et al. (2004; yellow: $M_{*}=1.59 M_{\odot}$ and $d=348 \mathrm{pc}$ ) and from Piétu et al. (2003; red: $M_{*}=2.1 M_{\odot}$ and $d=400 \mathrm{pc}$ ). The radial inner and outer extent of the curves is taken from the inner and outer radius of the emission listed in Table 3. We also overplot in both panels with gray vertical lines the radial distances of $0.34^{\prime \prime}$ and $0.63^{\prime \prime}$ to mark the band where the continuum emission is strongest.

The CO outer disk size derived in this work scaled to the stellar distance of Merín et al. (2004). For the $\mathrm{HCO}^{+} \mathrm{PV}$ diagrams we draw rotation curves between 85 au and an outer radius derived in this paper scaled the stellar distance (400 and $348 \mathrm{pc}$ ) in both papers.

These PV diagram helps illustrate two points. First, our choice for the closer distance of 325 au together with a lighter stellar mass is still in agreement with the CO rotation curves. Second, the $\mathrm{CO}$ emission is suppressed at the location where the continuum emission is strongest, between $0.34^{\prime \prime}$ and $0.63^{\prime \prime}$. This latter is an artifact of the data reduction, where subtracting the continuum emission suppresses line emission at those locations where the emission is optically thick or, in case of optically thin emission, where the dust in the midplane absorbs some emission coming from the far side of the disk.

We do not detect any kinematical deviation from Keplerian rotation in the $\mathrm{CO}$ emission within the gap as predicted for the interaction of Jupiter-mass planets with the gas disk (Perez et al. 2015b). The observations we present are of the highly optically thick ${ }^{12} \mathrm{CO} J=3-2$ transition and were made with both lower spatial and sensitivity compared to those predictions presented 
in Perez et al. (2015b). These three factors all conspire to make a possible kinematical imprint in the gas by the companion more difficult to detect. A deeper observation on an optically thinner isotopologue such as ${ }^{13} \mathrm{CO}$ at ALMA's highest resolution could help uncover a putative companion in HD 34282.

The $\mathrm{HCO}^{+} J=4-3$ emission is detected in closer proximity to the dust ring starting at the cavity outer radius and extending out to $1.3^{\prime \prime}$. Compared to the $\mathrm{CO}$ emission, the $\mathrm{HCO}^{+}$ $J=4-3$ emission originates closer to the midplane; this is very similar to the emission detected from the HD 97048 disk (van der Plas et al. 2017). Because $\mathrm{HCO}^{+}$ions quickly disappear without gas-phase CO molecules (e.g. Cleeves et al. 2014), we interpret this height difference as a vertical temperature gradient.

\subsection{Comparison of the radial extent of the dust and gas disk}

The radial extent of the disk as measured with ${ }^{12} \mathrm{CO} J=$ 3-2 emission is a factor of 2.7 larger than the disk measured at $345 \mathrm{GHz}$. While such a difference can be largely explained by different optical depths for the dust and gas (e.g. Dutrey et al. 1998; Guilloteau \& Dutrey 1998) without the need for radial drift of larger particles (Facchini et al. 2017), the sharp drop at $1.15^{\prime \prime}$ is very reminiscent of the drop seen in, for example, the disks around TW Hya (Hogerheijde et al. 2016; Andrews et al. 2016) and HD 97048 (van der Plas et al. 2017). We follow the interpretation in those works that the sharp drop is caused by the outer edge of a drift-dominated dust distribution (Birnstiel \& Andrews 2014; Facchini et al. 2017) and the tail end of the radial intensity distribution as a smearing effect by the beam of the outer disk edge at $1.15^{\prime \prime}$.

\section{Conclusions}

We resolve the disk around HD 34282 in the dust continuum emission at $867 \mu \mathrm{m}$ into a ring between $0.24^{\prime \prime}$ and $1.15^{\prime \prime}$ or between $78_{-11}^{+7}$ and $374_{-54}^{+33}$ au using a distance of $374_{-54}^{+33}$. There is an azimuthal asymmetry present in the dust continuum emission that coincides with the radial position of the ring but is radially unresolved, with an azimuthal extent of $52^{\circ}$, and contains $5 \%$ of the total sub-mm flux on top of the background disk emission. We also detect ${ }^{12} \mathrm{CO} J=3-2$ and $\mathrm{HCO}^{+} J=4-3$ emission lines. Assuming Keplerian rotation, we detect $\mathrm{CO}$ emission between 24 and $1000 \mathrm{au}, 2.7$ times as far out as the mm dust grains. The sharp outer edge of the dust disk suggests that this is due to radial drift.

We discount photoevaporation as an opening mechanism for the disk cavity. Rather, the disk cavity and azimuthal structure, the presence of gas within the cavity, and the low stellar accretion, all can be explained by the presence of $\mathrm{a} \approx 50 M_{\text {jup }}$ brown dwarf companion in the gap at a distance of $\approx 0.1^{\prime \prime}$.

Acknowledgements. We thank the referee for the comments which helped to improve the clarity of this paper. This paper makes use of data from ALMA programme 2013.1.00658.S. ALMA is a partnership of ESO (representing its member states), NSF (USA) and NINS (Japan), together with NRC (Canada) and NSC and ASIAA (Taiwan), in cooperation with the Republic of Chile. The Joint ALMA Observatory is operated by ESO, AUI/NRAO and NAOJ. The National Radio Astronomy Observatory is a facility of the National Science Foundation operated under cooperative agreement by Associated Universities, Inc. This work has made use of data from the European Space Agency (ESA) mission Gaia (http://www.cosmos.esa.int/gaia), processed by the Gaia Data Processing and Analysis Consortium (DPAC, http://www. cosmos.esa.int/web/gaia/dpac/consortium). Funding for the DPAC has been provided by national institutions, in particular the institutions participating in the Gaia Multilateral Agreement. G.v.d.P., C.C. and S.C acknowledge support from the Millennium Science Initiative (Chilean Ministry of Economy) through grant RC130007. G.P. acknowledges financial support from FONDECYT, grant 3140393, L.C. acknowledges support from FONDECYT grant 1171246, and S.C. acknowledges support from FONDECYT grant 1130949. G.P. and F.M. acknowledge funding from ANR of France under contract number ANR-16-CE31-0013 (Planet-Forming-Disks). C.C. acknowledges support from CONICYT PAI/Concurso nacional de insercion en la academia 2015, Folio 79150049. H.A. acknowledges the financial support of the Swiss National Science Foundation within the framework of the National Centre for Competence in Research Planets and H.C. acknowledges support from the Spanish grant AYA 2014-55840-P.

\section{References}

Acke, B., Min, M., van den Ancker, M. E., et al. 2009, A\&A, 502, L17 Alexander, R. D., Clarke, C. J., \& Pringle, J. E. 2006, MNRAS, 369, 216 ALMA Partnership, Brogan, C. L., Pérez, L. M., et al. 2015, ApJ, 808, L3 Amado, P. J., Moya, A., Suárez, J. C., et al. 2004, MNRAS, 352, L11 Andrews, S. M., Rosenfeld, K. A., Kraus, A. L., \& Wilner, D. J. 2013, ApJ, 771, 129

Andrews, S. M., Wilner, D. J., Zhu, Z., et al. 2016, ApJ, 820, L40

Artymowicz, P., \& Lubow, S. H. 1994, ApJ, 421, 651

Avenhaus, H., Quanz, S. P., Schmid, H. M., et al. 2014, ApJ, 781, 87

Baines, D., Oudmaijer, R. D., Porter, J. M., \& Pozzo, M. 2006, MNRAS, 367, 737

Bast, J. E., Brown, J. M., Herczeg, G. J., van Dishoeck, E. F., \& Pontoppidan, K. M. 2011, A\&A, 527, A119

Birnstiel, T., \& Andrews, S. M. 2014, ApJ, 780, 153

Brown, J. M., Blake, G. A., Qi, C., et al. 2009, ApJ, 704, 496

Canovas, H., Schreiber, M. R., Cáceres, C., et al. 2015, ApJ, 805, 21

Canovas, H., Caceres, C., Schreiber, M. R., et al. 2016, MNRAS, 458, L29

Carmona, A., van den Ancker, M. E., Thi, W.-F., Goto, M., \& Henning, T. 2005,

A\&A, 436, 977

Carmona, A., Pinte, C., Thi, W. F., et al. 2014, A\&A, 567, A51

Casassus, S., van der Plas, G., M, S. P., et al. 2013, Nature, 493, 191

Casey, M. P., Zwintz, K., Guenther, D. B., et al. 2013, MNRAS, 428, 2596

Clarke, C. J., Gendrin, A., \& Sotomayor, M. 2001, MNRAS, 328, 485

Cleeves, L. I., Bergin, E. A., \& Adams, F. C. 2014, ApJ, 794, 123

de Gregorio-Monsalvo, I., Ménard, F., Dent, W., et al. 2013, A\&A, 557, A133

de Val-Borro, M., Artymowicz, P., D’Angelo, G., \& Peplinski, A. 2007, A\&A, 471,1043

Draine, B. T., \& Lee, H. M. 1984, ApJ, 285, 89

Dullemond, C. P., \& Dominik, C. 2004, A\&A, 417, 159

Dutrey, A., Guilloteau, S., Prato, L., et al. 1998, A\&A, 338, L63

Dzyurkevich, N., Flock, M., Turner, N. J., Klahr, H., \& Henning, T. 2010, A\&A, 515, A70

Facchini, S., Birnstiel, T., Bruderer, S., \& van Dishoeck, E. F. 2017, A\&A, 605, A16

Fairlamb, J. R., Oudmaijer, R. D., Mendigutía, I., Ilee, J. D., \& van den Ancker,

M. E. 2015, MNRAS, 453, 976

Gaia Collaboration (Prusti, T., et al.) 2016, A\&A, 595, A1

Gaia Collaboration (Brown, A. G. A., et al.) 2016, A\&A, 595, A2

Garufi, A., Meeus, G., Benisty, M., et al. 2017, A\&A, 603, A21

Greaves, J. S., Mannings, V., \& Holland, W. S. 2000, Icarus, 143, 155

Gonzalez, J.-F., Laibe, G., \& Maddison, S. T. 2017, MNRAS, 467, 1984

Guilloteau, S., \& Dutrey, A. 1998, A\&A, 339, 467

Hildebrand, R. H. 1983, Quant. J. Roy. Astron. Soc., 24, 267

Högbom, J. A. 1974, A\&AS, 15, 417

Hogerheijde, M. R., Bekkers, D., Pinilla, P., et al. 2016, A\&A, 586, A99

Honda, M., Maaskant, K., Okamoto, Y. K., et al. 2012, ApJ, 752, 143

Isella, A., Guidi, G. Testi, L., et al. 2016, Phys. Rev. Lett., 117, 25

Kama, M., Folsom, C. P., \& Pinilla, P. 2015, A\&A, 582, L10

Khalafinejad, S., Maaskant, K. M., Mariñas, N., \& Tielens, A. G. G. M. 2016 , A\&A, 587, A62

Kraus, A. L., \& Ireland, M. J. 2012, ApJ, 745, 5

Laibe, G. 2014, MNRAS, 437, 3037

Laor, A., \& Draine, B. T. 1993, ApJ, 402, 441

Lovelace, R. V. E., Li, H., Colgate, S. A., \& Nelson, A. F. 1999, ApJ, 513, 805 Lyra, W., \& Lin, M.-K. 2013, ApJ, 775, 17

Maaskant, K. M., Honda, M., Waters, L. B. F. M., et al. 2013, A\&A, 555, A64 Maaskant, K. M., Min, M., Waters, L. B. F. M., \& Tielens, A. G. G. M. 2014 A\&A, 563, A78

Martí-Vidal, I., Vlemmings, W. H. T., Muller, S., \& Casey, S. 2014, A\&A, 563, A136

Meeus, G., Waters, L. B. F. M., Bouwman, J., et al. 2001, A\&A, 365, 476

Meheut, H., Meliani, Z., Varniere, P., \& Benz, W. 2012, A\&A, 545, A134

Merín, B., Montesinos, B., Eiroa, C., et al. 2004, A\&A, 419, 301 
McMullin, J. P., Waters, B., Schiebel, D., Young, W., \& Golap, K. 2007, Astronomical Data Analysis Software and Systems XVI, 376, 127

Natta, A., Testi, L., Neri, R., Shepherd, D. S., \& Wilner, D. J. 2004, A\&A, 416 179

Nomura, H., Tsukagoshi, T., Kawabe, R., et al. 2016, ApJ, 819, L7

Owen, J. E., Ercolano, B., Clarke, C. J., \& Alexander, R. D. 2010, MNRAS, 401, 1415

Pérez, L. M., Carpenter, J. M., Chandler, C. J., et al. 2012, ApJ, 760, L17

Perez, S., Casassus, S., Ménard, F., et al. 2015a, ApJ, 798, 85

Perez, S., Dunhill, A., Casassus, S., et al. 2015b, ApJ, 811, L5

Piétu, V., Dutrey, A., \& Kahane, C. 2003, A\&A, 398, 565

Pinilla, P., Benisty, M., \& Birnstiel, T. 2012, A\&A, 545, A81

Pinilla, P., van der Marel, N., Pérez, L. M., et al. 2015, A\&A, 584, A16

Pinte, C., Dent, W. R. F., Ménard, F., et al. 2016, ApJ, 816, 25

Pontoppidan, K. M., Blake, G. A., \& Smette, A. 2011, ApJ, 733, 84

Ragusa, E., Dipierro, G., Lodato, G., Laibe, G., \& Price, D. J. 2017, MNRAS, 464, 1449

Richer, J. S., \& Padman, R. 1991, MNRAS, 251, 707

Rosenfeld, K. A., Andrews, S. M., Hughes, A. M., Wilner, D. J., \& Qi, C. 2013, ApJ, 774, 16
Rosenfeld, K. A., Chiang, E., \& Andrews, S. M. 2014, ApJ, 782, 62

Testi, L., Birnstiel, T., Ricci, L., et al. 2014, Protostars and Planets VI (Tucson: University of Arizona Press), 339

Tsukagoshi, T., Nomura, H., Muto, T., et al. 2016, ApJ, 829, L35

Uribe, A. L., Klahr, H., Flock, M., \& Henning, T. 2011, ApJ, 736, 85

van der Marel, N., van Dishoeck, E. F., Bruderer, S., Pérez, L., \& Isella, A. 2015, A\&A, 579, A106

van der Plas, G., van den Ancker, M. E., Acke, B., et al. 2009, A\&A, 500, 1137 van der Plas, G., van den Ancker, M. E., Waters, L. B. F. M., \& Dominik, C. 2015, A\&A, 574, A75

van der Plas, G., Wright, C. M., Ménard, F., et al. 2017, A\&A, 597, A32

van Leeuwen, F. 2007, A\&A, 474, 653

Walsh, C., Juhász, A., Pinilla, P., et al. 2014, ApJ, 791, L6

Weidenschilling, S. J. 1977, MNRAS, 180, 57

Weingartner, J. C., \& Draine, B. T. 2000, BAAS, 32, 42.07

Wheelwright, H. E., Oudmaijer, R. D., \& Goodwin, S. P. 2010, MNRAS, 401, 1199

Williams, J. P., \& Cieza, L. A. 2011, ARA\&A, 49, 67

Woitke, P., Min, M., Pinte, C., et al. 2016, A\&A, 586, A103

Zhang, K., Bergin, E. A., Blake, G. A., et al. 2016, ApJ, 818, L16 